\title{
Historia de los dispositivos de administración de insulina
}

\section{History of insulin delivery devices}

\author{
ALDO FERrEIRA-HeRMOSILLO ${ }^{1 *}$ y DANIEL ELÍAS-LÓPEZ ${ }^{2}$ \\ 'Unidad de Investigación Médica en Enfermedades Endocrinas, Centro Médico Nacional Siglo XXI, Instituto Mexicano del Seguro \\ Social; ${ }^{2}$ Departamento de Endocrinología, Instituto Nacional de Ciencias Médicas y Nutrición Salvador Zubirán. Ciudad de México, \\ México
}

\section{RESUMEN}

En este artículo revisamos la historia de los dispositivos para aplicación de insulina, desde las jeringas de vidrio con agujas metálicas y esterilizables hasta la aparición de dispositivos en pluma, desechables y con agujas de pequeño calibre, así como las microinfusoras de insulina. Además, exploramos el desarrollo de insulinas administradas mediante vía oral e inhalada que han contado con la aprobación de la Food and Drug Administration o bien se han quedado en fase de ensayos clínicos por su baja biodisponibilidad, efectos adversos y reacciones secundarias o no han tenido difusión por su poco práctico dispositivo. Finalmente, comentamos otras vías de administración de la insulina y las limitaciones a las que se enfrentarán los nuevos dispositivos aun después de aprobados.

Palabras clave: Historia. Insulina. Diabetes mellitus. Tecnología biomédica.

\begin{abstract}
In this article we review the history of insulin delivery devices, from glass syringes with sterilizable metallic needles to the appearance of injector pens, disposable and with smallgauge needles, as well as continuous subcutaneous insulin infusion pumps. Furthermore, we explore the development of the oral and inhaled insulin which have been approved by the Food and Drug Administration or have been ceased due its low bioavailability, adverse effects, secondary reactions or lack of diffusion related with their non-practical device. Finally, we discuss other routes of insulin administration and the limitations that new devices will face even after its approval.
\end{abstract}

Key words: History. Insulin. Diabetes mellitus. Biomedical technology.

\section{Correspondencia:}

*Aldo Ferreira-Hermosillo

E-mail: aldo.nagisa@gmail.com
Fecha de recepción: 08-08-2021

Fecha de aceptación: 18-10-2021 DOI: 10.24875/RME.M21000016
Disponible en internet: 05-11-2021 RevMexEndocrinol Metab Nutr.2021;8:(SUPL.3):29-37

2462-4144 / @ 2021 Sociedad Mexicana de Nutrición y Endocrinología, AC. Publicado por Permanyer. Este es un artículo open access bajo la licencia CC BY-NC-ND (http://creativecommons.org/licenses/by-nc-nd/4.0/). 


\section{INTRODUCCIÓN}

No cabe duda de que la historia de la insulina es una de las más bellas en la medicina'. Más allá de toda la ciencia que ha implicado su aislamiento, síntesis farmacológica utilizando tecnología de ADN recombinante, modificaciones químicas que han alargado y aplanado su efecto por más de 24 horas ( $y$ ahora incluso una insulina de uso semanal ${ }^{2}$ ) o disminuido su pico de acción y duración para el control de la glucosa posprandial (con el desarrollo de las insulinas ultrarrápidas ${ }^{3}$ ), resulta fascinante la historia «detrás de cámaras»: las controversias, frustraciones, sueños e ilusiones relacionadas con su descubrimiento. Por ejemplo, ¿qué hubiera pasado si Eugène Gley hubiera publicado su descubrimiento de la disminución de glucosuria del extracto acuoso del páncreas en perros con síntomas de diabetes desde 1890?, o bien ¿qué consecuencias hubiese tenido que el joven Banting hubiese preferido su carrera militar en lugar de continuar con sus investigaciones en el laboratorio de MacLeod? ${ }^{4}$. Pequeños cambios en la historia que hubiesen impactado para bien o para mal en la vida de millones de personas para quienes hoy en día la insulina es el tratamiento principal para el control de su glucosa.

Evidentemente, junto con la evolución de la insulina debían evolucionar también los dispositivos para su aplicación. En un inicio la insulina era almacenada y comercializada en viales de cristal (situación que increíblemente prevalece, un siglo después) y la única vía para su administración eran jeringas de vidrio que tenían que ser esterilizadas entre cada uso. Por si fuera poco, las agujas para su aplicación eran de un calibre grueso, de gran tamaño y después de ciertas aplicaciones requerían ser afiladas utilizando una piedra pómez $z^{5}$. Las aplicaciones eran intramusculares, dos veces por día y los volúmenes de aplicación eran de 5 a $18 \mathrm{ml}^{6}$. Treinta años después, en 1955, aparecieron las primeras jeringas de plástico (Ilamadas Monoject) y para mediados de 1960 ya existían las jeringas para insulina U100, marcadas en $\mathrm{UI}$, de 0.3, 0.5 y $1 \mathrm{ml}(30,50 \text { y } 100 \mathrm{U})^{6}$.

Para 1963, el Dr. Arnold Kadisch, de Los Ángeles (California), diseñó la primera bomba de insulina y glucagón que, debido a su tamaño, nunca fue comercializada. No fue hasta finales de los 70 cuando empezaron los primeros intentos de crear dispositivos prácticos que infundieran insulina subcutánea de forma continua y en 1986 MiniMed introdujo su primer modelo: el 502, dando paso a la creación de infusoras cada vez más pequeñas, más fáciles de manejar y con el advenimiento de la tecnología inalámbrica, menos estorbosas y más acopladas al día a día del paciente que vive con diabetes ${ }^{4}$. Por si fuera poco, las infusoras se han acoplado con el monitoreo continuo de glucosa, en un intento (cada vez más palpable) de «cerrar el ciclo»y crear un «páncreas artificial».

En 1985, Novo Nordisk comercializó la primera pluma de insulina (llamada NovoPen), un dispositivo compacto, discreto, con un cartucho desechable, una aguja más pequeña y la posibilidad de administrar un click. No encuentro una mejor descripción del hito que representó este descubrimiento para los pacientes con diabetes que lo relatado por Paolo Di Bartolo y Robert Eckel (dos endocrinólogos viviendo con diabetes): «Recordamos con emoción la presentación de este nuevo método de administración de insulina, un regalo sorprendente que cambió nuestra vida. No más jeringas, botellas, dependencia del refrigerador $u$ otras formas de mantener fría la insulina. Seríamos libres para salir, viajar, decidir a último momento el no regresar a casa para comer o cenar: lo único que necesitaríamos sería sacar nuestra pluma del bolsillo o bolsa» ${ }^{7}$.

Desde entonces, también la tecnología de las plumas ha avanzado; de tal forma que hoy tenemos dispositivos más delgados, con botones de aplicación más suaves, agujas más pequeñas, finas y lubricadas, cuyo objetivo es disminuir el área y dolor a la aplicación. Desafortunadamente en nuestro país las plumas no son ampliamente utilizadas, debido a que no forman parte del cuadro básico de las instituciones públicas, mientras que en otros países como EE.UU. su uso se ha visto limitado por la falta de cobertura del seguro médico ${ }^{6}$.

Finalmente, también en los años 70 aparecieron los primeros dispositivos libres de agujas como MediJect o Injex, que requerían un adaptador para llenar el cartucho desde el vial, preparación especial del dispositivo (cebado) y mayor presión para la 
aplicación del medicamento; además, el primer dispositivo era poco amigable y de mayor tamaño. Debido a estas características, adicional al alto costo, muy pocos fueron comercializados y perdieron popularidad rápidamente ${ }^{6}$.

Tal como puede observarse, el objetivo siguió siendo desarrollar un sistema de aplicación de insulina sencillo, cómodo e indoloro, por lo que el siguiente paso lógico fue considerar el uso de insulina inhalada o por vía oral.

INSULINA ORAL: BARRERAS INICIALES PARA SU DESARROLLO

El 30-50\% de los pacientes que utilizan insulina reportan ansiedad o miedo durante su aplicación relacionado con el uso de jeringas y agujas ${ }^{8}$; esto impacta en la adherencia a su tratamiento, altera su habilidad de administración y complica su control glucémico. Es por esto por lo que surge la necesidad de buscar nuevas vías de administración como la oral o nasal.

Además del beneficio evidente de ser una vía indolora, el uso oral mejora los niveles de insulina portal y limita la hiperinsulinemia periférica, lo que disminuye la ganancia de peso $y$, debido al aumento del metabolismo hepático, disminuye el riesgo de hipoglucemias y la reacción inmunitaria generada por la administración subcutánea ${ }^{9}$. A pesar de parecer una alternativa ideal, las primeras limitantes en el desarrollo de la insulina oral provienen de su propia naturaleza proteica; así, su biodisponibilidad es baja debido a los siguientes factores ${ }^{10}$ :

- Enzimas proteolíticas. El intestino delgado es el principal sitio de absorción de los aminoácidos y las proteínas se enfrentan a degradación por enzimas como pepsina, tripsina, quimiotripsina y carboxipeptidasas; por lo que el primer reto para el desarrollo de la insulina oral fue elaborar un vehículo que protegiera a la proteína activa y permitiera que llegase a su destino final en el íleo.

- Alto peso molecular. La insulina tiene un peso molecular de 5,800 Da, por lo que su absorción por la membrana mucosa columnar se ve limitado.
Por si fuera poco, el tracto gastrointestinal (Gl) posee una capa hidrofílica y otra hidrofóbica, que obstaculiza la absorción de la insulina (hidrofílica). La única opción para vencer esta barrera es aprovechar la absorción paracelular, por lo que había que tomar en cuenta para su desarrollo la presencia de uniones celulares estrechas.

- Capa mucosa. Constituye una barrera para la absorción de diferentes proteínas, incluyendo la insulina.

Estas barreras han podido abordarse gracias al avance tecnológico y a la experiencia con otras proteínas de uso terapéutico. Por ejemplo, agregando ácido cítrico a las tabletas de calcitonina se favorece el transporte paracelular y se inhiben las proteasas intestinales. Otras estrategias han sido: el uso de inhibidores enzimáticos (como el glucocolato de sodio, mesilato de camostat, bacitracina o el ovomucoide de pato), cuya principal limitante es la inhibición de las propias enzimas Gl y la malabsorción; el uso de potenciadores de la absorción no tóxicos, farmacológica y químicamente inertes, no irritantes y no alergénicos (como ZOT), con la limitante de que al aumentar demasiado la permeabilidad se puede dar paso hacia la circulación sistémica a toxinas y bacterias, sobre todo cuando hay compromiso del epitelio intestinal; el uso de modificadores químicos para aumentar la absorción mediada por receptores específicos (como la conjugación con transferrina), cuya desventaja es que pueden ser tan efectivos que producen efecto de depósito y efecto hipoglucemiante retardado; y el uso de polímeros mucoadhesivos (como el quitosano o el ácido láctico poliglicólico), con la desventaja de retrasar tanto la absorción, que se aumente la exposición a las enzimas proteolíticas. Por si fuera poco, el desarrollo de la formulación en tabletas se enfrenta a que diferentes aminoácidos de la insulina son susceptibles a oxidación, desaminación e hidrólisis, así como a cambios en su estabilidad física generados por la agregación molecular, las variaciones en temperatura, el pH y la degradación enzimática una vez que se alcanza la circulación sistémica ${ }^{10}$.

Debido a todas estas limitantes, son pocas las insulinas orales que han llegado a ser probadas en ensayos clínicos. 


\section{MÁS DE 15 AÑOS DE INVESTIGACIÓN} EN EL DESARROLLO DE INSULINA ORAL

En 2007, la compañía Empisphere Technologies (EE. UU.) registró la patente de una técnica para el desarrollo de insulina oral (Eligen), mediante la combinación de granulación seca y húmeda. La tableta contenía 300 unidades de insulina y $160 \mathrm{mg}$ del acarreador 4-CNAB (N-4 cloro saliciloil 4-aminobutirato monosódico) que aumentaba la absorción intestinal, sin alterar la estructura de la molécula. La tableta tenía un inicio de acción de 30 minutos y duración de 1 hora, por lo que su indicación era el control de glucosa posprandial; sin embargo, en un estudio clínico usándola por dos semanas existieron amplias variaciones en la absorción y restringieron su uso clínico.

La compañía canadiense Generex Biotechnology produjo el aerosol Oral-lyn para uso preprandial en pacientes con diabetes mellitus tipo 1 (DM1) o tipo 2 (DM2). Se encuentra en investigación en ensayos clínicos fase III en Canadá, EE.UU. y Europa, y desde septiembre de 2008 la Food and Drug Administration (FDA) lo consideró dentro del programa de «Nuevos Medicamentos en Investigación», que se refiere a que se permite su uso en aquellos que no fueron candidatos a participar en los estudios clínicos y para los que no hay otra alternativa satisfactoria. Su uso en Ecuador y Líbano ya fue aprobado. Su formulación incluye potenciadores de la absorción y surfactantes que permiten la formación de micelas, las cuales son administradas por medio del dispositivo RapidMist. Debido al tamaño de las micelas liberadas (mayor a $7 \mu \mathrm{m}$ ), la insulina se queda en la boca y no alcanza la vía aérea. Cada cartucho contiene $400 \mathrm{U}$ de insulina y cada disparo libera $10 \mathrm{U}$, pero debido a que solo se absorbe un $10 \%$, cada disparo implica $1 \mathrm{U}$ de insulina que alcanzará la circulación, lo cual limita su uso y dificulta su apego a largo plazo. La ventaja de su uso radica en su rápida absorción (10 minutos), alcanzando su pico a los 30 minutos y regresando a niveles basales a los 150 minutos $^{11}$.

En India en 2010 surgió la tecnología Nobex, que combinó una modificación química de la insulina
(IN-105) con polietilenglicol y un potenciador de la absorción (caproato de sodio); siendo desarrollada posteriormente en forma de tabletas por Biocon. La IN-105 o tregopil es un análogo de acción rápida, que a las dosis de 10, 15, 20 y $30 \mathrm{mg}$ redujo la glucosa en un 18, 26.1, 29 y $30.8 \%{ }^{10}$. En los estudios fase III, el $25 \%$ de los participantes (20 sujetos con DM2 e índice de masa corporal [IMC] $<29 \mathrm{~kg} / \mathrm{m}^{2}$ ) presentaron hipoglucemia $(<70 \mathrm{mg} / \mathrm{dl})$ dentro de los 30-60 minutos de su toma, así como elevación de los triglicéridos séricos, mareo e hiperhidrosis. Además, en un estudio fase III a seis meses no logró cumplir con el objetivo primario de disminución de hemoglobina glucosilada ( $\mathrm{HbA} 1 \mathrm{c})$ del $0.7 \%$ en comparación con placebo. El último estudio sobre esta insulina fue en 2020, donde se evaluó el efecto en la excursión de la glucosa posprandial en un rango de 10-20 minutos antes de un alimento y con un intervalo de cinco horas entre alimentos ${ }^{9}$.

En 2015, Novo Nordisk y Merrion Pharmaceuticals desarrollaron tecnología para incrementar la permeabilidad $\mathrm{Gl}$ en un intento de desarrollar un análogo de insulina de acción prolongada, llamado GIPET I, que se encuentra en estudios clínicos fase II. La misma tecnología está utilizándose para sintetizar otros dos análogos: insulina 287 e insulina 320 (ambos en fase I) ${ }^{11}$. Finalmente, en 2019 Novo Nordisk publicó los resultados de la insulina O8388, utilizando caproato de sodio como potenciador de la absorción; tiene unión reversible a la albúmina por medio de la adición de un ácido graso de 18 carbonos y tiene baja afinidad por el receptor, permitiendo una vida media de aproximadamente 70 horas. En un ensayo clínico fase lla con uso diario una vez al día por ocho semanas mostró seguridad y eficacia similar al uso de glargina U100; sin embargo, su baja biodisponibilidad provocó que fuera descontinuada.

Finamente, en 2016 Oramed Pharmaceuticals fabricó la formulación oral ORMD-0801, una cápsula con cubierta entérica junto con inhibidores de proteasas y potenciadores de la absorción. Las cápsulas se toman tres veces al día, 45 minutos antes de las comidas y en los estudios fase llb se observó disminución de la $\mathrm{HbA} 1 \mathrm{c}$ del $1.0 \%$ después de 12 semanas de su uso en pacientes con DM2; además en otro estudio se observó disminución de la 
gammaglutamil transpeptidasa. Los estudios fase III iniciaron a finales del $2020^{9}$.

Otros productos como Capsulin (insulina 150 o 300 U con una cápsula de absorción entérica), están en estudios fase $1 I^{11}$.

\section{ADMINISTRACIÓN DE INSULINA PULMONAR}

Junto con la administración de la insulina oral, surgieron estudios para el desarrollo de insulinas que pudieran absorberse por vía pulmonar. Sin embargo, solo dos han logrado aprobación por la FDA y la European Medicines Agency (EMA): Exubera y Afrezza ${ }^{11}$.

Exubera fue aprobada en 2006, después de demostrar no inferioridad en términos de eficacia (disminución de HbA1c) en pacientes con DM1 o con DM2 en comparación con insulina NPH (Neutral Protamine Hagedorn)/rápida. Estaba indicada como terapia para combinación con insulina de acción prolongada en DM1 y como monoterapia o en combinación con insulina basal o medicamentos orales en DM2. Se contraindicó en pacientes fumadores activos o con antecedente de tabaquismo en los últimos seis meses, ya que esto aumentaba el riesgo de hipoglucemia. Debido a que los cambios en la función pulmonar afectaban su absorción y aumentaban el riesgo de hipoglucemia o hiperglucemia, también estaba contraindicada en pacientes con asma o enfermedad pulmonar obstructiva crónica ${ }^{5}$.

En la práctica clínica, el uso de Exubera se asoció a aumento en el riesgo de infección respiratoria, tos, faringitis y rinitis. Además, debido a que en los estudios clínicos se observó que disminuía la función pulmonar durante su uso, se recomendó la evaluación mediante espirometría antes de iniciarlo y en intervalos regulares, lo cual dificultó su recomendación. Por otra parte, debido al diseño de su dispositivo, era difícil de cargar y poco discreto, requería entrenamiento para su uso y era costoso ${ }^{12}$.

Por si fuera poco, existió una advertencia de seguridad después de que se reportaran seis casos de cáncer pulmonar en comparación con un caso en el grupo control. Si bien no se demostró correlación directa con el uso de Exubera, esta situación generó desconfianza en pacientes y médicos. Por todas estas situaciones Pfizer decidió retirarla del mercado en $2007^{5}$.

Afrezza fue aprobada por la FDA en 2014. Su eficacia y seguridad fue demostrada en los estudios AFFINITY. En el AFFINITY-1 se observó que, en combinación con insulina basal, el uso de Afrezza no era inferior a aspart para la disminución de $\mathrm{HbA} 1 \mathrm{c}$ en pacientes con DM1 y que existía mejoría en el número de hipoglucemias ${ }^{13}$. En el estudio AFFINITY-2 se confirmó que el uso de Afrezza era eficaz para la disminución de $\mathrm{HbA} 1 \mathrm{c}$ en pacientes con DM2 inadecuadamente controlados con antidiabéticos orales, pero existía un mayor número de hipoglucemias al compararse con insulina rápida ${ }^{14}$. En comparación con Exubera, su dispositivo de aplicación es pequeño, ligero y dosificado en unidades $(4,8 \mathrm{y}$ $12 \mathrm{U}$ ). Después de su inhalación, las partículas de insulina Tecnosphere (con un tamaño de 2-2.5 $\mu \mathrm{m}$ ) son aerosolizadas y llevadas a los alvéolos, donde se disuelven gracias al líquido alveolar ${ }^{15}$. En los estudios en pacientes con DM1 su inicio de acción fue a los 12 minutos, el pico ocurrió a los 35 a 55 minutos y se regresaba a los niveles basales a los 90-270 minutos después de su administración ${ }^{16}$.

Su uso no se recomienda para pacientes con enfermedad pulmonar crónica por el riesgo de broncoespasmo agudo, ni en aquellos fumadores o que recientemente dejaron de fumar ${ }^{11}$.

Con respecto al cáncer pulmonar, durante los estudios clínicos se reportaron dos casos en 2,750 pacientes/año estudiados en pacientes fumadores que utilizaron Afrezza; al término de los estudios otros dos casos de carcinoma de células escamosas fueron reportados en no fumadores. No se reportaron carcinomas en el grupo control. Si bien los estudios en modelos murinos han demostrado que Afrezza no tiene efectos oncogénicos, no hay suficientes estudios al respecto en seres humanos. Otro efecto adverso común es la tos, presente en el $28 \%$ de los pacientes usuarios de esta insulina, en comparación con el $4.4 \%$ reportado en los grupos control. En los estudios clínicos también se observó disminución del volumen espiratorio forzado (VEF1) 
en los primeros tres meses de su uso y hasta el $6 \%$ tuvieron una disminución $>15 \%$ por hasta dos $a_{n}{ }^{5}{ }^{5}$. Adicionalmente, el inserto incluyó que en los estudios se observó una incidencia 207\% mayor de cetoacidosis diabética en pacientes con DM1.

Otro producto que se encuentra en estudio es la insulina líquida en aerosol Dance-501 de Aerogen. Este tipo de insulina llega hasta tejido alveolar, provoca menos tos y es más económica; sin embargo, depende de una capacitación inicial adecuada al ser aspirable y de que el paciente ponga la cantidad adecuada en el inhalador, lo que podría ser un problema para pacientes mayores o con alguna limitación física. Hasta el momento, los estudios han demostrado que no hay diferencias en su biodisponibilidad y biopotencia en comparación con la insulina subcutánea (aunque requiere una dosificación 10 veces mayor) y que su absorción es más rápida ${ }^{17}$.

\section{OTROS DISPOSITIVOS DE ADMINISTRACIÓN}

La tecnología inalámbrica ha permitido la evolución de otros métodos de aplicación de insulina, los denominados «bomba en parche», que no requieren tubos para su aplicación.

Omnipod (Insulet Corporation) es un dispositivo disponible en EE.UU., Reino Unido, Alemania e Israel. Fue aprobado por la FDA en 2005 y para el 2010 en el resto de los países. El dispositivo está formado por un reservorio de $200 \mathrm{U}$ de insulina, que la libera por medio de una aguja discreta, y un dispositivo de manejo personal que usa tecnología inalámbrica para dosificar la insulina basal e incorpora un monitor de glucosa. El dispositivo debe ser llenado con insulina por el paciente y puede continuar adherido hasta por 72 horas $^{18}$.

Otro dispositivo que salió a la venta desde 2012 es V-Go (Valeritas), que cubre hasta por 24 horas la administración de insulina basal o los bolos y no requiere baterías, programación o manejo remoto. Se carga por medio de un adaptador con insulina lispro o aspart y se inserta en la piel del abdomen, brazos o muslos por medio de una aguja móvil de $4.6 \mathrm{~mm}$. Los basales están predeterminados en 20, 30 o $40 \mathrm{U}$ y se puede administrar hasta $36 \mathrm{U}$ de insulina como bolos, presionando un botón a la hora de las comidas.

Otros sistemas como JewelPUMP (Debiotech) incorporan un sistema microelectromecánico desechable junto con el reservorio cuando está vacío, con una carcasa que contiene baterías, control, monitoreo y alarma; de igual forma el sistema Cellnovo (Cellnovo), es «semidesechable» y comprende la bomba con un cartucho desechable controlado por el teléfono que «sube» datos a una página web en tiempo real ${ }^{6}$.

Finalmente, se ha desarrollado un parche de administración transdérmica que usa frecuencias de ultrasonido especiales para abrir los poros de la piel y empujar la insulina a través de la dermis hasta la circulación sistémica sin utilizar agujas Ilamado UStrip (Transdermal Specialities), que puede utilizarse en pacientes con DM1 o DM2. El aparato es portátil, operado con baterías y diseñado para liberar insulina lispro en la piel de brazos o abdomen por medio de un programa manejado por una pantalla táctil. Actualmente se encuentra en estudios clínicos fase III.

En la tabla 1 se resumen los dispositivos para la administración de insulina, señalando sus principales ventajas y desventajas.

\section{LOS RETOS A LOS QUE SE ENFRENTAN LOS NUEVOS DISPOSITIVOS DE ADMINISTRACIÓN}

A pesar de que han existido a lo largo de la historia diferentes dispositivos para la administración de insulina, varios han sido descontinuados u olvidados. Esto no tiene relación con su tecnología o posible aplicabilidad (ya que el objetivo primario es aumentar la adherencia al tratamiento con insulina), y en la mayoría de los casos más bien obedece a cuestiones de ventas: no se le dio la difusión suficiente entre médicos y pacientes, sus costos no son cubiertos por las aseguradoras médicas y el precio a mediano y largo plazo es mayor al de las tecnologías 
Tabla 1. Resumen de los dispositivos para administración de insulina

\begin{tabular}{|c|c|c|}
\hline Tipo de dispositivo & Ventajas & Desventajas \\
\hline Jeringas para insulina & $\begin{array}{l}\text { Bajo costo } \\
\text { Diferentes capacidades dependiendo de la } \\
\text { dosis de insulina ( } 30,50 \text { y } 100 \mathrm{U}) \\
\text { Diferentes tamaños de aguja }(6,8 \text { y } 13 \mathrm{~mm}) \\
\text { Permiten mezclar algunas insulinas (p. ej., } \\
\text { NPH y rápida) }\end{array}$ & $\begin{array}{l}\text { Destinadas para uso de insulina en viales, se } \\
\text { requiere que el paciente tenga un } \\
\text { entrenamiento sobre cómo sacar la insulina } \\
\text { La dosis puede variar dependiendo de la } \\
\text { adecuada titulación del paciente (uso } \\
\text { complicado en pacientes con falla visual) } \\
\text { Mayor dolor en sitio de aplicación } \\
\text { Puede generar reacciones locales en el sitio de } \\
\text { aplicación } \\
\text { No permite transportar la insulina }\end{array}$ \\
\hline Plumas para insulina & $\begin{array}{l}\text { Permiten transportar insulina sin necesidad } \\
\text { de refrigeración } \\
\text { Aplicación exacta de la dosis indicada } \\
\text { Agujas de menor tamaño (4, 5, } 6 \text { y } 8 \mathrm{~mm}) \\
\text { Cada giro en el dispositivo es una unidad } \\
\text { (audible). Pueden usarse en personas con } \\
\text { dificultad visual }\end{array}$ & $\begin{array}{l}\text { Costo mayor que las jeringas } \\
\text { No permite la mezcla de insulinas } \\
\text { Algunas requieren que se deseche un poco } \\
\text { de insulina de forma inicial } \\
\text { Puede generar reacciones locales en el sitio } \\
\text { de aplicación }\end{array}$ \\
\hline Microinfusoras de insulina & $\begin{array}{l}\text { Se puede aplicar insulina basal y en bolos } \\
\text { con el mismo dispositivo } \\
\text { Permite incrementos de dosis de hasta } \\
0.025 \text { U/hora con diferentes horarios y } \\
\text { ajustar diferentes sensibilidades y patrones } \\
\text { de aplicación (días de enfermedad, basales } \\
\text { temporales para ejercicio) } \\
\text { Con el uso integrado de un sensor de } \\
\text { glucosa, disminuye el riesgo de } \\
\text { hipoglucemias e hiperglucemias }\end{array}$ & $\begin{array}{l}\text { Costo más elevado } \\
\text { Puede resultar incómodo para algunos } \\
\text { pacientes portar el dispositivo } \\
\text { La disfunción mecánica u oclusión del } \\
\text { mecanismo de suministro provoca } \\
\text { rápidamente hiperglucemia }\end{array}$ \\
\hline Insulina oral & $\begin{array}{l}\text { Comodidad al evitar la aplicación vía } \\
\text { jeringa } \\
\text { Disminuye hiperinsulinemia periférica } \\
\text { (menor ganancia de peso y riesgo de } \\
\text { hipoglucemias) } \\
\text { No genera reacciones en sitio de aplicación } \\
\text { ¿Mayor adherencia?* }\end{array}$ & $\begin{array}{l}\text { Problemas para la absorción y degradación } \\
\text { por enzimas proteolíticas propias del tracto Gl } \\
\text { Uso limitado al control de glucosa } \\
\text { posprandial } \\
\text { ¿Costo?* }\end{array}$ \\
\hline Insulina inhalada & $\begin{array}{l}\text { Comodidad al evitar la aplicación vía } \\
\text { jeringa } \\
\text { Dispositivo de aplicación pequeño, ligero y } \\
\text { dosificado en } 4,8 \text { y } 12 \mathrm{U} \\
\text { ¿Mayor adherencia?* }\end{array}$ & $\begin{array}{l}\text { Contraindicada en pacientes con tabaquismo } \\
\text { o enfermedades pulmonares } \\
\text { Generan tos, algunos estudios reportan } \\
\text { disminución del VEF1 } \\
\text { Uso limitado al control de glucosa } \\
\text { posprandial }\end{array}$ \\
\hline Bomba en parche & $\begin{array}{l}\text { A diferencia de las microinfusoras, no } \\
\text { requieren tubos para la aplicación de } \\
\text { insulina. } \\
\text { Dosificación con dispositivo inalámbrico } \\
\text { (mayor discreción). }\end{array}$ & $\begin{array}{l}\text { Debe ser llenado con insulina en intervalos } \\
\text { regulares } \\
\text { Algunos dispositivos tienen basales } \\
\text { predeterminados (no ajustables), lo que no } \\
\text { permite dosis individualizadas }\end{array}$ \\
\hline
\end{tabular}

NPH: Neutral Protamine Hagedorn; GI: gastrointestinal; VEF1: volumen espiratorio forzado.

*No existen estudios sobre las características señaladas.

actuales, no se pueden conseguir fácilmente, o bien los dispositivos son confusos, y poco prácticos y, más que ayudar, complican la vida del paciente ${ }^{6}$.
Por último, el avance de la tecnología ha hecho que la competencia se vuelva más difícil, ya existen: plumas desechables con aplicadores que cuantifican 
las dosis, se comunican con dispositivos periféricos (teléfono celular) con programas que se acoplan al monitoreo de glucosa o permiten la administración de insulina basal y prandial; aquellas que se combinan con análogos del péptido similar al glucagón tipo 1 y que solo requieren una aplicación; las que se aplican de forma semanal, y el desarrollo de microinfusoras que han evolucionado hasta casi lograr «cerrar el circuito». Es claro que un nuevo dispositivo tendrá que demostrar además de su eficacia y seguridad que puede sobrevivir ante la gran cantidad de opciones con las que (afortunadamente) cuenta el paciente que vive con diabetes hoy en día.

\section{CONCLUSIÓN}

Uno de los principales retos en el tratamiento de los pacientes que viven con diabetes es mejorar la adherencia al tratamiento más eficaz: la insulina. En la práctica diaria, todos nos hemos enfrentado al obstáculo que representa su administración por medio de una inyección. Influye el miedo al dolor, la percepción de que es complicado administrársela y en ocasiones la propia enfermedad genera discapacidad para su uso correcto. Así como la molécula ha evolucionado a lo largo de la historia, también lo han hecho los dispositivos para su uso. Hoy nos resulta difícil pensar que un paciente se inyecte con una jeringa de $100 \mathrm{U}$ con agujas de $13 \mathrm{~mm}$ (y sin embargo, sucede), gracias a que se han hecho más populares las insulinas en pluma, las cuales son desechables y fáciles de usar. Si bien en la actualidad la aplicación inyectable es menos dolorosa y más práctica, aún existe la necesidad de explorar otras vías como la inhalada, oral o peritoneal, para minimizar la incomodidad generada en nuestros pacientes.

En este artículo revisamos la historia de estos dispositivos, las investigaciones que se han hecho para conseguir su aprobación, sus ventajas y desventajas y por qué muchos no están disponibles. Consideramos que, debido a sus características, una forma de administración que tiene un gran potencial es la vía oral; sin embargo, parece que aún faltan algunos años para tenerla disponible. Esperemos que la tecnología nos siga asombrando y que en un futuro existan más opciones que permitan mejorar la aceptación y apego al tratamiento con insulina $y$, por ende, la disminución de complicaciones y mejoría de la calidad de vida de los pacientes que viven con diabetes.

\section{AGRADECIMIENTOS}

Los autores agradecen a la mesa directiva de la Sociedad Mexicana de Nutrición y Endocrinología, así como a los coordinadores de este suplemento por la invitación para escribir este artículo.

\section{FINANCIAMIENTO}

La presente investigación no ha recibido ninguna beca específica de agencias de los sectores públicos, comercial o con ánimo de lucro.

CONFLICTO DE INTERESES

Los autores declaran no tener conflicto de intereses.

\section{RESPONSABILIDADES ÉTICAS}

Protección de personas y animales. Los autores declaran que para esta investigación no se han realizado experimentos en seres humanos ni en animales.

Confidencialidad de los datos. Los autores declaran que en este artículo no aparecen datos de pacientes.

Derecho a la privacidad y consentimiento informado. Los autores declaran que en este artículo no aparecen datos de pacientes. 


\section{BIBLIOGRAFÍA}

1. Hegele RA, Maltman GM. Insulin's centenary: the birth of an idea. Lancet Diabetes Endocrinol. 2020;8(12):971-7.

2. Rosenstock J, Bajaj HS, Janez A, Silver R, Begtrup K, Hansen MV, et al. Once-weekly insulin for type 2 diabetes without previous insulin treatment. N Engl J Med. 2020;383(22):2107-16.

3. Haahr $\mathrm{H}$, Heise $\mathrm{T}$. Fast-acting insulin aspart: A review of its pharmacokinetic and pharmacodynamic properties and the clinical consequences. Clin Pharmacokinet. 2020;59(2):155-72.

4. Vecchio I, Tornali C, Bragazzi NL, Martini M. The discovery of insulin: An important milestone in the history of medicine. Front Endocrinol (Lausanne). 2018;9:613.

5. Oleck J, Kassam S, Goldman JD. Commentary: Why was inhaled insulin a failure in the market? Diabetes Spectr. 2016;29(3):180-4.

6. Fry A. Insulin delivery device technology 2012: where are we after 90 years? J Diabetes Sci Technol. 2012;6(4):947-53.

7. Di Bartolo $P$, Eckel RH. Living with insulin: The story of insulin from people with diabetes. Diabetes Res Clin Pract. 2021;176:108857.

8. Kruger DF, LaRue S, Estepa P. Recognition of and steps to mitigate anxiety and fear of pain in injectable diabetes treatment. Diabetes Metab Syndr Obes. 2015;8:49-56.

9. Kurtzhals P, Nishimura E, Haahr H, Hoeg-Jensen T, Johansson E, Madsen $P$, et al. Commemorating insulin's centennial: engineering insulin pharmacology towards physiology. Trends Pharmacol Sci. 2021;42(8):620-39.
10. Wong CY, Martinez J, Dass CR. Oral delivery of insulin for treatment of diabetes: status quo, challenges and opportunities. J Pharm Pharmacol. 2016;68(9):1093-108.

11. Easa N, Alany RG, Carew M, Vangala A. A review of non-invasive insulin delivery systems for diabetes therapy in clinical trials over the past decade. Drug Discov Today. 2019;24(2):440-51.

12. Heinemann $\mathrm{L}$. The failure of exubera: are we beating a dead horse? Diabetes Sci Technol. 2008;2(3):518-29.

13. Bode BW, McGill JB, Lorber DL, Gross JL, Chang PC, Bregman DB, et al. Inhaled technosphere insulin compared with injected prandial insulin in type 1 diabetes: A randomized 24-week trial. Diabetes Care. 2015;38(12):2266-73.

14. Rosenstock J, Franco D, Korpachev V, Shumel B, Ma Y, Baughman R, et al. Inhaled technosphere insulin versus inhaled technosphere placebo in insulin-naive subjects with type 2 diabetes inadequately controlled on oral antidiabetes agents. Diabetes Care. 2015;38(12): 2274-81.

15. Kim ES, Plosker GL. AFREZZA(R) (insulin human) inhalation powder: A review in diabetes mellitus. Drugs. 2015;75(14):1679-86.

16. Heinemann L, Parkin CG. Rethinking the viability and utility of inhaled insulin in clinical practice. J Diabetes Res. 2018;2018:4568903.

17. Fink JB, Molloy L, Patton JS, Galindo-Filho VC, de Melo Barcelar J, Alcoforado $\mathrm{L}$, et al. Good things in small packages: an innovative delivery approach for inhaled insulin. Pharm Res. 2017;34(12): 2568-78.

18. Zisser HC. The OmniPod Insulin Management System: the latest innovation in insulin pump therapy. Diabetes Ther. 2010;1(1): $10-24$ 